Antonio Espuña, Moisès Graells and Luis Puigjaner (Editors), Proceedings of the $27^{\text {th }}$ European Symposium on Computer Aided Process Engineering - ESCAPE 27

October $1^{\text {st }}-5^{\text {th }}, 2017$, Barcelona, Spain (C) 2017 Elsevier B.V. All rights reserved.

\title{
Global Identification of Kinetic Parameters via the Extent-based Incremental Approach
}

\author{
Diogo Rodrigues*, Julien Billeter, Dominique Bonvin \\ Laboratoire d'Automatique, EPFL, 1015 Lausanne, Switzerland \\ diogo.mateusrodrigues@epfl.ch
}

\begin{abstract}
This contribution presents a kinetic model identification scheme that guarantees convergence to global optimality. The use of the extent-based incremental approach allows one to (i) identify each reaction individually, and (ii) reduce the number of parameters to identify via optimization to the ones that appear nonlinearly in the investigated rate law. Via Taylor expansion, the identification problem can be rearranged as a polynomial optimization problem with coefficients computed only once prior to optimization. The optimization problem is then reformulated as a convex optimization problem, namely a semidefinite program, which converges to global optimality. The approach is demonstrated via a simulated example.
\end{abstract}

Keywords: global optimization, parameter estimation, model identification, vessel extents.

\section{Introduction}

The identification of reaction kinetics represents the main challenge in building models for reaction systems. The identification task can be performed via a simultaneous or an incremental approach. In simultaneous model identification, a rate law is postulated for each reaction, and all rate parameters are estimated simultaneously, which often leads to slow and difficult convergence due to the large number of model parameters. This procedure is repeated for all combinations of rate candidates, which makes the approach computationally expensive. In extent-based incremental model identification, each reaction is dealt with individually. Consequently, for one reaction at a time, only the rate candidates for that reaction need to be compared, which requires estimating only the parameters of a given rate candidate (Bhatt et al., 2012). The measured concentrations are first transformed to experimental extents (Rodrigues et al., 2015), and then the rate laws are identified individually by comparing the experimental extents with the modeled extents that result from integration of the candidate rate laws.

Most parameter estimation methods only enforce local optimality, which may result in an incorrect model. The incremental approach is suited to global optimization since each estimation sub-problem involves only a small set of parameters. This paper presents an extension to extent-based incremental model identification that guarantees global optimality by solving a semidefinite program that results from the reformulation of a polynomial optimization problem with constant coefficients (Lasserre, 2001).

\section{Extent-based incremental model identification}

This section reviews the fundamental features of extent-based incremental model identification.

\subsection{Rate law}

As mentioned in the introduction, the extent-based incremental approach deals with one rate candidate at a time. The rate candidates for a given reaction correspond to plausible rate laws, 
thus involving selected combinations of parameters that can only take values in a finite set. Hence, each optimization problem in this paper considers a single rate law of known structure, with the decision variables being limited to the parameters that can take any value in the set of real numbers.

Let us assume that the reaction rate law $r$ is a function of the $S$-dimensional vector of concentrations $\mathbf{c}(t)$. Furthermore, $r$ is also linear in the $L$ parameters $\boldsymbol{\alpha}=\left(\alpha_{1}, \ldots, \alpha_{L}\right)$ and nonlinear in the $N$ parameters $\boldsymbol{\theta}=\left(\theta_{1}, \ldots, \theta_{N}\right)$, which can be exploited to simplify the treatment of the parameters $\boldsymbol{\alpha}$, as will be shown in the sequel. Hence, the reaction rate is expressed as

$r(\mathbf{c}(t), \boldsymbol{\alpha}, \boldsymbol{\theta})=r_{0}(\mathbf{c}(t), \boldsymbol{\theta})+\sum_{\ell=1}^{L} \alpha_{\ell} r_{\ell}(\mathbf{c}(t), \boldsymbol{\theta})$.

The goal of the method is to estimate the rate parameters $\boldsymbol{\alpha}$ and $\boldsymbol{\theta}$ from measured data.

\subsection{Identification problem}

Considering the $i$ th reaction rate as the rate $r$ in Eq. (1), the $i$ th vessel extent of reaction is (Amrhein et al., 2010)

$x_{r, i}(t, \boldsymbol{\alpha}, \boldsymbol{\theta})=\int_{0}^{t} V(\tau) r(\mathbf{c}(\tau), \boldsymbol{\alpha}, \boldsymbol{\theta}) e^{-\int_{\tau}^{t} \omega(\zeta) \mathrm{d} \zeta} \mathrm{d} \tau=V(t) d_{0}(t, \boldsymbol{\theta})+\sum_{\ell=1}^{L} \alpha_{\ell} V(t) d_{\ell}(t, \boldsymbol{\theta})$,

where $V(t)$ and $\omega(t)$ are the volume and the inverse of the residence time, and $d_{\ell}(t, \boldsymbol{\theta}):=$ $\int_{0}^{t} \frac{V(\tau)}{V(t)} r_{\ell}(\mathbf{c}(\tau), \boldsymbol{\theta}) e^{-\int_{\tau}^{t} \omega(\zeta) \mathrm{d} \zeta} \mathrm{d} \tau, \forall \ell=0, \ldots, L$

In practice, the noisy measurements $\tilde{\mathbf{c}}$ are available only at the time instants $t_{h}:=h T$, for $h=0, \ldots, H$. Then, upon numerical integration and replacing $r_{\ell}\left(\mathbf{c}\left(t_{h}\right), \boldsymbol{\theta}\right)$ by its estimate $\hat{r}_{\ell}\left(\tilde{\mathbf{c}}\left(t_{h}\right), \boldsymbol{\theta}\right), d_{\ell}\left(t_{h}, \boldsymbol{\theta}\right)$ is approximated by $\hat{d}_{\ell}\left(t_{h}, \boldsymbol{\theta}\right), \forall \ell=0, \ldots, L$.

Assuming each element of $\tilde{\mathbf{c}}(t)$ is corrupted by i.i.d. noise, the identification problem reads

$\min _{\boldsymbol{\alpha}, \boldsymbol{\theta}} J(\boldsymbol{\alpha}, \boldsymbol{\theta})=\sum_{h=1}^{H} \frac{1}{H}\left(\frac{\hat{x}_{r, i}\left(t_{h}, \boldsymbol{\alpha}, \boldsymbol{\theta}\right)-\tilde{x}_{r, i}\left(t_{h}\right)}{V\left(t_{h}\right)}\right)^{2}$,

with the modeled extent $\hat{x}_{r, i}\left(t_{h}, \boldsymbol{\alpha}, \boldsymbol{\theta}\right):=V\left(t_{h}\right) \hat{d}_{0}\left(t_{h}, \boldsymbol{\theta}\right)+\sum_{\ell=1}^{L} \alpha_{\ell} V\left(t_{h}\right) \hat{d}_{\ell}\left(t_{h}, \boldsymbol{\theta}\right)$ linear in $\boldsymbol{\alpha}$, and the experimental extent $\tilde{x}_{r, i}\left(t_{h}\right)$ given by the linear transformation of the measurements $\tilde{\mathbf{c}}\left(t_{h}\right)$

$\tilde{\mathbf{x}}_{r}\left(t_{h}\right)=V\left(t_{h}\right) \mathscr{T}_{r}\left(\tilde{\mathbf{c}}\left(t_{h}\right)-\mathbf{W}_{i n} \frac{\mathbf{x}_{i n}\left(t_{h}\right)}{V\left(t_{h}\right)}-V_{0} \mathbf{c}_{0} \frac{x_{i c}\left(t_{h}\right)}{V\left(t_{h}\right)}\right)$

where $\mathscr{T}_{r}$ is constructed such that $\mathscr{T}_{r} \mathbf{N}^{\mathrm{T}}=\mathbf{I}_{R}$, with the $R \times S$ stoichiometric matrix $\mathbf{N}$, and $\mathbf{W}_{\text {in }}$ is the $S \times p$ inlet-composition matrix, with $R$ and $p$ the numbers of independent reactions and independent inlets, respectively. The extents of inlet $\mathbf{x}_{i n}$ and of initial conditions $x_{i c}$ can be computed from the knowledge of inlet and outlet flowrates (Rodrigues et al., 2015).

\subsection{Reformulation of the identification problem}

The cost function in Eq. (3) is quadratic in $\boldsymbol{\alpha}$, that is, $J(\boldsymbol{\alpha}, \boldsymbol{\theta})=c(\boldsymbol{\theta})+2 \boldsymbol{\alpha}^{\mathrm{T}} \mathbf{g}(\boldsymbol{\theta})+\boldsymbol{\alpha}^{\mathrm{T}} \mathbf{H}(\boldsymbol{\theta}) \boldsymbol{\alpha}$. Then, since the optimal parameters $\boldsymbol{\alpha}$ can be computed for each $\boldsymbol{\theta}$ as

$\boldsymbol{\alpha}^{*}(\boldsymbol{\theta})=-\mathbf{H}(\boldsymbol{\theta})^{-1} \mathbf{g}(\boldsymbol{\theta})$,

the problem in Eq. (3) can be reformulated as a problem with only the decision variables $\boldsymbol{\theta}$,

$\min _{\boldsymbol{\theta}} \bar{J}(\boldsymbol{\theta})=J\left(\boldsymbol{\alpha}^{*}(\boldsymbol{\theta}), \boldsymbol{\theta}\right)=c(\boldsymbol{\theta})-\mathbf{g}(\boldsymbol{\theta})^{\mathrm{T}} \mathbf{H}(\boldsymbol{\theta})^{-1} \mathbf{g}(\boldsymbol{\theta})$. 
One can also compute $\frac{\partial \bar{J}}{\partial \theta_{k}}(\boldsymbol{\theta}), \forall k=1, \ldots, N$, analytically to speed up convergence of the nonlinear optimization algorithm. However, the optimization problem in Eq. (6) may have several local minima. Furthermore, the functions $\hat{d}_{0}(t, \boldsymbol{\theta}), \ldots, \hat{d}_{L}(t, \boldsymbol{\theta})$ need to be computed at each iteration, since $\boldsymbol{\theta}$ varies.

\section{Convex extent-based incremental model identification}

This section presents a method that solves the identification problem to global optimality without requiring function evaluation at each iteration.

\subsection{Taylor series expansion of the rate law}

Let us consider the rate law given in Section 2. The rate $r$ can be written as a multivariate Taylor series if $r_{0}, \ldots, r_{L}$ are infinitely differentiable functions and there exists a vector $\overline{\boldsymbol{\theta}}$ and a set $\mathscr{P}$ such that the Taylor series converges $\forall \boldsymbol{\Delta} \boldsymbol{\theta} \in \mathscr{P}$, that is,

$r(\mathbf{c}(t), \boldsymbol{\alpha}, \boldsymbol{\theta})=\lim _{n \rightarrow \infty} \sum_{\mathbf{k} \in \mathscr{K}_{n}}\left(\frac{1}{\mathbf{k} !} \frac{\partial^{\mathbf{k}} r_{0}}{\partial \boldsymbol{\theta}^{\mathbf{k}}}(\mathbf{c}(t), \overline{\boldsymbol{\theta}})+\sum_{\ell=1}^{L} \alpha_{\ell} \frac{1}{\mathbf{k} !} \frac{\partial^{\mathbf{k}} r_{\ell}}{\partial \boldsymbol{\theta}^{\mathbf{k}}}(\mathbf{c}(t), \overline{\boldsymbol{\theta}})\right) \boldsymbol{\Delta} \boldsymbol{\theta}^{\mathbf{k}}, \quad \forall \boldsymbol{\Delta} \boldsymbol{\theta} \in \mathscr{P}$,

where $\boldsymbol{\Delta} \boldsymbol{\theta}:=\boldsymbol{\theta}-\overline{\boldsymbol{\theta}}$ is the deviation of $\boldsymbol{\theta}$ around $\overline{\boldsymbol{\theta}}, \mathbf{k}:=\left(k_{1}, \ldots, k_{N}\right)$ is the vector of powers of a monomial, $\mathscr{K}_{n}:=\left\{\left(k_{1}, \ldots, k_{N}\right) \in \mathbb{N}_{0}^{N}: 0 \leq k_{1}+\ldots+k_{N} \leq n\right\}$ in the case of a polynomial of degree $n, \mathbf{k} !:=k_{1} ! \ldots k_{N} !, \boldsymbol{\Delta} \boldsymbol{\theta}^{\mathbf{k}}:=\left(\theta_{1}-\bar{\theta}_{1}\right)^{k_{1}} \ldots\left(\theta_{N}-\bar{\theta}_{N}\right)^{k_{N}}$ and $\frac{\partial^{\mathbf{k}}}{\partial \boldsymbol{\theta}^{\mathbf{k}}}:=\frac{\partial^{k_{1}+\ldots+k_{N}}}{\partial \theta_{1}^{k_{1}} \ldots \partial \theta_{N}^{k_{N}}}$.

\subsection{Approximate identification problem}

From Eqs. (2) and (7), the ith vessel extent of reaction is

$x_{r, i}(t, \boldsymbol{\alpha}, \boldsymbol{\theta})=\lim _{n \rightarrow \infty} \sum_{\mathbf{k} \in \mathscr{K}_{n}} V(t) d_{0, \mathbf{k}}(t) \boldsymbol{\Delta} \boldsymbol{\theta}^{\mathbf{k}}+\sum_{\ell=1}^{L} \alpha_{\ell} \sum_{\mathbf{k} \in \mathscr{K}_{n}} V(t) d_{\ell, \mathbf{k}}(t) \boldsymbol{\Delta} \boldsymbol{\theta}^{\mathbf{k}}, \quad \forall \boldsymbol{\Delta} \boldsymbol{\theta} \in \mathscr{P}$,

where $d_{\ell, \mathbf{k}}(t):=\int_{0}^{t} \frac{V(\tau)}{V(t)} \frac{1}{\mathbf{k} !} \frac{\partial^{\mathbf{k}} r_{\ell}}{\partial \boldsymbol{\theta}^{\mathbf{k}}}(\mathbf{c}(\tau), \overline{\boldsymbol{\theta}}) e^{-\int_{\tau}^{t} \omega(\zeta) \mathrm{d} \zeta} \mathrm{d} \tau, \forall \ell=0, \ldots, L, \forall \mathbf{k} \in \mathscr{K}_{n}$.

In practice, by integrating numerically and replacing $\frac{\partial^{\mathbf{k}} r_{\ell}}{\partial \boldsymbol{\theta}^{\mathbf{k}}}\left(\mathbf{c}\left(t_{h}\right), \overline{\boldsymbol{\theta}}\right)$ by its estimate $\hat{f}_{\ell, \mathbf{k}}\left(\tilde{\mathbf{c}}\left(t_{h}\right)\right)$, $d_{\ell, \mathbf{k}}\left(t_{h}\right)$ is approximated by $\hat{d}_{\ell, \mathbf{k}}\left(t_{h}\right), \forall \ell=0, \ldots, L, \forall \mathbf{k} \in \mathscr{K}_{n}$.

This leads to the identification problem

$\min _{\boldsymbol{\alpha}, \boldsymbol{\Delta} \boldsymbol{\theta}} J_{c}(\boldsymbol{\alpha}, \boldsymbol{\Delta} \boldsymbol{\theta})=\sum_{h=1}^{H} \frac{1}{H}\left(\frac{\hat{x}_{r, i}\left(t_{h}, \boldsymbol{\alpha}, \boldsymbol{\Delta} \boldsymbol{\theta}\right)-\tilde{x}_{r, i}\left(t_{h}\right)}{V\left(t_{h}\right)}\right)^{2}$,

with $\hat{x}_{r, i}\left(t_{h}, \boldsymbol{\alpha}, \boldsymbol{\Delta} \boldsymbol{\theta}\right):=\sum_{\mathbf{k} \in \mathscr{K}_{n}} V\left(t_{h}\right) \hat{d}_{0, \mathbf{k}}\left(t_{h}\right) \boldsymbol{\Delta} \boldsymbol{\theta}^{\mathbf{k}}+\sum_{\ell=1}^{L} \alpha_{\ell} \sum_{\mathbf{k} \in \mathscr{K}_{n}} V\left(t_{h}\right) \hat{d}_{\ell, \mathbf{k}}\left(t_{h}\right) \boldsymbol{\Delta} \boldsymbol{\theta}^{\mathbf{k}}$ for finite $n$.

\subsection{Reformulation as a polynomial optimization problem}

The cost function in Eq. (9) is quadratic in $\boldsymbol{\alpha}$, that is, $J_{c}(\boldsymbol{\alpha}, \boldsymbol{\Delta} \boldsymbol{\theta})=c_{c}(\boldsymbol{\Delta} \boldsymbol{\theta})+2 \boldsymbol{\alpha}^{\mathrm{T}} \mathbf{g}_{c}(\boldsymbol{\Delta} \boldsymbol{\theta})+$ $\boldsymbol{\alpha}^{\mathrm{T}} \mathbf{H}_{c}(\boldsymbol{\Delta} \boldsymbol{\theta}) \boldsymbol{\alpha}$, where the elements of $c_{c}(\boldsymbol{\Delta} \boldsymbol{\theta}), \mathbf{g}_{c}(\boldsymbol{\Delta} \boldsymbol{\theta})$ and $\mathbf{H}_{c}(\boldsymbol{\Delta} \boldsymbol{\theta})$ are polynomials of degree $2 n$ in $\boldsymbol{\Delta} \boldsymbol{\theta}$ with coefficients computed analytically from $\hat{d}_{0, \mathbf{k}}\left(t_{h}\right), \ldots, \hat{d}_{L, \mathbf{k}}\left(t_{h}\right)$ and $\frac{\tilde{x}_{r, i}\left(t_{h}\right)}{V\left(t_{h}\right)}$.

Since the optimal parameters $\boldsymbol{\alpha}$ can be computed for each $\boldsymbol{\Delta} \boldsymbol{\theta}$ as

$\boldsymbol{\alpha}_{c}^{*}(\boldsymbol{\Delta} \boldsymbol{\theta})=-\mathbf{H}_{c}(\boldsymbol{\Delta} \boldsymbol{\theta})^{-1} \mathbf{g}_{c}(\boldsymbol{\Delta} \boldsymbol{\theta})$,

the problem in Eq. (9) can be reformulated as a problem with only the decision variables $\boldsymbol{\Delta} \boldsymbol{\theta}$,

$\min _{\boldsymbol{\Delta} \boldsymbol{\theta}} \bar{J}_{c}(\boldsymbol{\Delta} \boldsymbol{\theta})=J_{c}\left(\boldsymbol{\alpha}_{c}^{*}(\boldsymbol{\Delta} \boldsymbol{\theta}), \boldsymbol{\Delta} \boldsymbol{\theta}\right)=c_{c}(\boldsymbol{\Delta} \boldsymbol{\theta})-\mathbf{g}_{c}(\boldsymbol{\Delta} \boldsymbol{\theta})^{\mathrm{T}} \mathbf{H}_{c}(\boldsymbol{\Delta} \boldsymbol{\theta})^{-1} \mathbf{g}_{c}(\boldsymbol{\Delta} \boldsymbol{\theta})=\frac{\operatorname{det}(\mathbf{M}(\boldsymbol{\Delta} \boldsymbol{\theta}))}{\operatorname{det}\left(\mathbf{H}_{c}(\boldsymbol{\Delta} \boldsymbol{\theta})\right)}$ 
where $\mathbf{M}(\boldsymbol{\Delta} \boldsymbol{\theta}):=\left[\begin{array}{ll}c_{c}(\boldsymbol{\Delta} \boldsymbol{\theta}) & \mathbf{g}_{c}(\boldsymbol{\Delta} \boldsymbol{\theta})^{\mathrm{T}} \\ \mathbf{g}_{c}(\boldsymbol{\Delta} \boldsymbol{\theta}) & \mathbf{H}_{c}(\boldsymbol{\Delta} \boldsymbol{\theta})\end{array}\right]$, and $P_{a}(\boldsymbol{\Delta} \boldsymbol{\theta}):=\operatorname{det}\left(\mathbf{H}_{c}(\boldsymbol{\Delta} \boldsymbol{\theta})\right)$ and $P_{b}(\boldsymbol{\Delta} \boldsymbol{\theta}):=\operatorname{det}(\mathbf{M}(\boldsymbol{\Delta} \boldsymbol{\theta}))$ are polynomials in $\boldsymbol{\Delta} \boldsymbol{\theta}$. The reformulation of $\bar{J}_{c}(\boldsymbol{\Delta} \boldsymbol{\theta})$ as a rational function is possible since it is the determinant of the Schur complement of $\mathbf{H}_{c}(\boldsymbol{\Delta} \boldsymbol{\theta})$ in $\mathbf{M}(\boldsymbol{\Delta} \boldsymbol{\theta})$ (Boyd and Vandenberghe, 2004). One can then write the problem in Eq. (11) as the polynomial optimization problem

$\max _{\tau} \tau \quad$ s.t. $\quad P_{b}(\boldsymbol{\Delta} \boldsymbol{\theta})-P_{a}(\boldsymbol{\Delta} \boldsymbol{\theta}) \tau \geq 0, \forall \boldsymbol{\Delta} \boldsymbol{\theta}$.

Since the coefficients of $P_{a}(\boldsymbol{\Delta} \boldsymbol{\theta})$ and $P_{b}(\boldsymbol{\Delta} \boldsymbol{\theta})$ do not depend on $\boldsymbol{\Delta} \boldsymbol{\theta}$, they do not have to be computed at each iteration. Hence, the problem in Eq. (12) is an algebraic estimation problem.

\subsection{Reformulation as a convex optimization problem}

Let us define $a_{\mathbf{k}}$ and $b_{\mathbf{k}}$ as the coefficients of $P_{a}(\boldsymbol{\Delta} \boldsymbol{\theta})$ and $P_{b}(\boldsymbol{\Delta} \boldsymbol{\theta})$ such that $P_{a}(\boldsymbol{\Delta} \boldsymbol{\theta})=$ $\sum_{\mathbf{k} \in \mathscr{K}_{2 d}} a_{\mathbf{k}} \boldsymbol{\Delta} \boldsymbol{\theta}^{\mathbf{k}}$ and $P_{b}(\boldsymbol{\Delta} \boldsymbol{\theta})=\sum_{\mathbf{k} \in \mathscr{K}_{2 d}} b_{\mathbf{k}} \boldsymbol{\Delta} \boldsymbol{\theta}^{\mathbf{k}}$, with $d \geq n(L+1)$, and assume that the optimal $\boldsymbol{\Delta} \boldsymbol{\theta}$ is in a compact set $\mathscr{C}=\left\{\mathbf{y}: \sum_{\mathbf{q} \in \mathscr{K}_{2}} c_{\mathbf{q}} \mathbf{y}^{\mathbf{q}} \geq 0\right\}$. By using the equivalence of nonnegative polynomials and conical combination of sum-of-squares polynomials on a compact set (Lasserre, 2001), the optimization problem in Eq. (12) can be written as the convex semidefinite program (SDP)

$\max _{\tau, \mathbf{Q}_{0}, \mathbf{Q}_{1}}$

$$
\begin{array}{ll}
\text { s.t. } & \mathbf{Q}_{0} \succeq \mathbf{0}_{s(N, d) \times s(N, d)} \\
& \mathbf{Q}_{1} \succeq \mathbf{0}_{s(N, d-1) \times s(N, d-1)} \\
& b_{\mathbf{k}}-a_{\mathbf{k}} \tau=\operatorname{tr}\left(\mathbf{R}_{0, \mathbf{k}} \mathbf{Q}_{0}\right)+\sum_{\substack{\mathbf{q} \in \mathscr{K}_{2} \\
\mathbf{k}-\mathbf{q} \in \mathscr{K}_{2 d}}} c_{\mathbf{q}} \operatorname{tr}\left(\mathbf{R}_{1, \mathbf{k}-\mathbf{q}} \mathbf{Q}_{1}\right), \quad \forall \mathbf{k} \in \mathscr{K}_{2 d},
\end{array}
$$

where $s(N, d):=\left(\begin{array}{c}N+d \\ d\end{array}\right)$, and $\mathbf{R}_{0, \mathbf{k}}$ and $\mathbf{R}_{1, \mathbf{k}}$ are localizing matrices such that $\sum_{\mathbf{k} \in \mathscr{K}_{2 d}} \mathbf{R}_{0, \mathbf{k}} \boldsymbol{\Delta} \boldsymbol{\theta}^{\mathbf{k}}=$ $\mathbf{v}_{d}(\boldsymbol{\Delta} \boldsymbol{\theta}) \mathbf{v}_{d}(\boldsymbol{\Delta} \boldsymbol{\theta})^{\mathrm{T}}$ and $\sum_{\mathbf{k} \in \mathscr{K}_{2 d}} \mathbf{R}_{1, \mathbf{k}} \boldsymbol{\Delta} \boldsymbol{\theta} \mathbf{k}^{\mathbf{k}}=\mathbf{v}_{d-1}(\boldsymbol{\Delta} \boldsymbol{\theta}) \mathbf{v}_{d-1}(\boldsymbol{\Delta} \boldsymbol{\theta})^{\mathrm{T}}$, with the $s(N, d)$-dimensional vector of monomials up to degree $d$ in the $N$ variables $\boldsymbol{\Delta} \boldsymbol{\theta}$ defined as $\mathbf{v}_{d}(\boldsymbol{\Delta} \boldsymbol{\theta})$.

This SDP is constrained by a linear matrix inequality (LMI) of size $s(N, d)$ and another of size $s(N, d-1)$. However, one expects a small problem size, since the numbers $L$ and $N$ of model parameters are usually low in the incremental approach. The degree $n$ of the Taylor series should be large enough to allow a good approximation of the rate $r$, but not too large, since otherwise the size of the SDP would grow too much and the matrices that describe it would become ill-conditioned.

Note that the optimization problems in Eqs. (12) and (13) are equivalent with $d=n(L+1)$ if $P_{a}(\boldsymbol{\Delta} \boldsymbol{\theta})$ and $P_{b}(\boldsymbol{\Delta} \boldsymbol{\theta})$ are univariate polynomials $(N=1)$. In the case of multivariate polynomials $(N \geq 2)$, the equivalence between these optimization problems holds only for some $d \geq n(L+1)$ such that $\operatorname{rank}\left(\sum_{\mathbf{k} \in \mathscr{K}_{2 d}} \mathbf{R}_{0, \mathbf{k}} \mu_{\mathbf{k}}\right)=\operatorname{rank}\left(\sum_{\mathbf{k} \in \mathscr{K}_{2 d}} \mathbf{R}_{1, \mathbf{k}} \mu_{\mathbf{k}}\right)$, where $\mu_{\mathbf{k}}$ is the dual variable of the equality constraint in Eq. (13), $\forall \mathbf{k} \in \mathscr{K}_{2 d}$ (Lasserre, 2009).

\subsection{Computing solutions}

The SDP in Eq. (13) yields the minimum $\bar{J}_{c}\left(\boldsymbol{\Delta} \boldsymbol{\theta}^{*}\right)=\tau^{*}$, but it does not provide an explicit way of finding the global solution $\boldsymbol{\Delta} \boldsymbol{\theta}^{*}$ (and thus $\boldsymbol{\alpha}^{*}$ ).

For numerical reasons, the solutions to the primal and dual problems should be combined to obtain the best solution $\boldsymbol{\Delta} \boldsymbol{\theta}^{*}$. For the primal problem, $\mathbf{v}_{d}\left(\boldsymbol{\Delta} \boldsymbol{\theta}^{*}\right)$ lies in the null space of $\mathbf{Q}_{0}^{*}$, whereas for the dual problem, $\mathbf{v}_{d}\left(\boldsymbol{\Delta} \boldsymbol{\theta}^{*}\right)$ lies in the row space of $\mathbf{L}_{0}^{*}$, where $\mathbf{L}_{0}$ is the dual variable of the LMI $\mathbf{Q}_{0} \succeq \mathbf{0}_{s(N, d) \times s(N, d)}$ in Eq. (13). An algorithm that computes the solutions $\boldsymbol{\Delta} \boldsymbol{\theta}^{*}$, using the knowledge of the space where $\mathbf{v}_{d}\left(\boldsymbol{\Delta} \boldsymbol{\theta}^{*}\right)$ lies, is described by Lasserre (2009).

Finally, the optimal values $\boldsymbol{\alpha}^{*}=\boldsymbol{\alpha}_{c}^{*}\left(\boldsymbol{\Delta} \boldsymbol{\theta}^{*}\right)$ can be computed according to Eq. (10). 


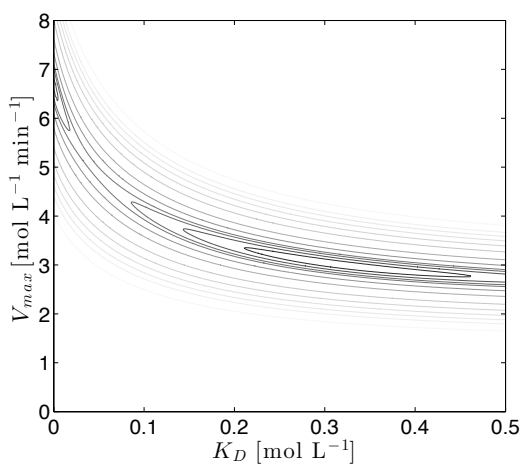

Figure 1: Contour plot of the cost function $J\left(V_{\max }, K_{D}\right)$. The contour lines are shown with increasingly lighter shading for $J\left(V_{\max }, K_{D}\right) \in\{0.03,0.09,0.17,0.25,0.5,1,2,3,4,5,6,8,10\}$.

\section{Simulated example}

This section presents the example of an extent-based incremental model identification problem for which more than one local minimum exists. This example consists in the identification of the maximum rate $V_{\max }$ and inhibition constant $K_{D}$ of the enzymatic decomposition $\mathrm{S} \rightarrow 2 \mathrm{I}$ in a batch reactor. The reaction $\mathrm{I} \rightarrow \mathrm{P}$ also takes place in the reactor. The concentrations of $\mathrm{S}, \mathrm{I}$ and $\mathrm{P}$ are denoted as $c_{S}, c_{I}$ and $c_{P}$, and $\mathbf{c}=\left[\begin{array}{lll}c_{S} & c_{I} & c_{P}\end{array}\right]^{\mathrm{T}}$. The stoichiometry is given by $\mathbf{N}=\left[\begin{array}{ccc}-1 & 2 & 0 \\ 0 & -1 & 1\end{array}\right]$. The kinetics of the first reaction results from the model of an enzyme with two binding sites of equal binding affinity, no cooperativity, and previously known substrate inhibition (Lin et al., 2001). The dynamics of $c_{S}$ is described by

$\dot{c}_{S}=-r(\mathbf{c}, \alpha, \theta), \quad c_{S}(0)=2 \mathrm{~mol} \mathrm{~L}^{-1}$,

with

$r(\mathbf{c}, \alpha, \theta)=\alpha \frac{\frac{c_{S}}{\theta}+0.1 \frac{c_{S}^{2}}{\theta^{2}}}{1+2 \frac{c_{S}}{\theta}+\frac{c_{S}^{2}}{\theta^{2}}}$,

where $\alpha=V_{\text {max }}=3 \mathrm{~mol} \mathrm{~L}^{-1} \min ^{-1}$ and $\theta=K_{D}=0.32 \mathrm{~mol} \mathrm{~L}-1$, that is, $L=1$ and $N=1$.

Let us assume that an experiment is run for $3 \mathrm{~min}$ and noise-free measurements of the concentration $c_{S}$ are obtained at the sampling interval of $5 \mathrm{~s}$. For these data, a contour plot of the cost function $J\left(V_{\max }, K_{D}\right)$ can be drawn, as shown in Figure 1, which shows that two local minima exist. Depending on the initial guess, a regular optimization algorithm may not converge to the correct values of the parameters $V_{\max }$ and $K_{D}$. For example, a regular optimization algorithm with user-supplied gradients and using the initial guess $K_{D}=0.04 \mathrm{~mol} \mathrm{~L}^{-1}$ yields the solution $V_{\max }^{*}=6.63 \mathrm{~mol} \mathrm{~L}^{-1} \min ^{-1}, K_{D}^{*}=0.001 \mathrm{~mol} \mathrm{~L}^{-1}$, with $J\left(V_{\max }^{*}, K_{D}^{*}\right)=0.0702$. However, the convex optimization algorithm, using $n=20$ and $\bar{\theta}=1 \mathrm{~mol} \mathrm{~L}^{-1}$, yields the correct solution $V_{\max }^{*}=3 \mathrm{~mol} \mathrm{~L}{ }^{-1} \min ^{-1}, K_{D}^{*}=0.32 \mathrm{~mol} \mathrm{~L}^{-1}$, with $J\left(V_{\max }^{*}, K_{D}^{*}\right)=4.4 \times 10^{-9}$. Figure 2 shows that the fitted curves that result from these two solutions are clearly different, and only the convex algorithm predicts a concentration profile that matches the measured data.

\section{Conclusions}

This paper has shown that extent-based incremental model identification can be used to converge quickly to global optimality. Several features of extent-based incremental model identification contribute to this result. The cost function that results from this approach 


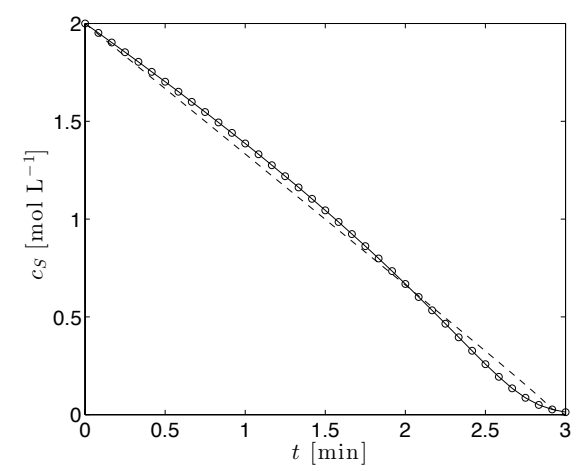

Figure 2: Measured concentration (circles) and fitting profiles that result from the solution to the identification problem using regular (dashed line) and convex (solid line) optimization algorithms.

involves only the parameters of a single rate candidate and is a quadratic function of the parameters in which the rate expression is linear. Then, this cost function can be converted via a Taylor series expansion into a rational function of the parameters in which a single rate expression is nonlinear. This rational function is used in the formulation of the identification problem as a polynomial optimization problem with constant coefficients computed prior to optimization. Finally, this polynomial optimization problem can be converted to an SDP, which can be handled by SDP solvers that efficiently attain global solutions upon convergence.

Consequently, guaranteed convergence to global optimality exists for virtually all identification problems in reaction systems, provided that some mild technical conditions are satisfied. For many of these problems, it would be practically infeasible to obtain global optimality via the standard simultaneous approach, due to the large number of model parameters and combinations of rate candidates. As shown by the simulated example in this paper, identification problems with more than one local minimum exist, and regular optimization algorithms may converge to a local minimum that is not the global one, whereas the proposed convex formulation guarantees convergence to the global minimum.

It is known that simultaneous model identification yields statistically optimal parameter estimators in the maximum-likelihood sense. These estimators are consistent, that is, they converge to the true values of the parameters as the number of data points tends to infinity, and have an acceptable quality in many practical situations (Bard, 1974). Hence, the next step in future work is to show that extent-based incremental model identification not only converges to global optimality, but can also be used to provide parameter estimates with similar quality.

\section{References}

M. Amrhein, N. Bhatt, B. Srinivasan, D. Bonvin, 2010, Extents of reaction and flow for homogeneous reaction systems with inlet and outlet streams, AIChE J., 56, 11, 2873-2886.

Y. Y. Bard, 1974. Nonlinear Parameter Estimation. Academic Press, New York.

N. Bhatt, N. Kerimoglu, M. Amrhein, W. Marquardt, D. Bonvin, 2012, Incremental identification for reaction systems - A comparison between rate-based and extent-based approaches, Chem. Eng. Sci., 83, 24-38.

S. Boyd, L. Vandenberghe, 2004. Convex Optimization. Cambridge University Press.

J. B. Lasserre, 2001, Global optimization with polynomials and the problem of moments, SIAM J. Optim., 11, 3, 796-817.

J. B. Lasserre, 2009. Moments, Positive Polynomials and Their Applications. World Scientific.

Y. Lin, P. Lu, C. Tang, Q. Mei, G. Sandig, A. D. Rodrigues, T. H. Rushmore, M. Shou, 2001, Substrate inhibition kinetics for cytochrome p450-catalyzed reactions, Drug Metabolism and Disposition, 29, 4, 368-374.

D. Rodrigues, S. Srinivasan, J. Billeter, D. Bonvin, 2015, Variant and invariant states for reaction systems, Comp. Chem. Eng., 73, 1, 23-33. 